Supplement of Biogeosciences, 15, 2309-2323, 2018

https://doi.org/10.5194/bg-15-2309-2018-supplement

(C) Author(s) 2018. This work is distributed under

the Creative Commons Attribution 4.0 License.

(c) (1)

Supplement of

\title{
Mercury distribution and transport in the North Atlantic Ocean along the GEOTRACES-GA01 transect
}

Daniel Cossa et al.

Correspondence to: Daniel Cossa (dcossa@ifremer.fr)

The copyright of individual parts of the supplement might differ from the CC BY 4.0 License. 
1. Supplementary figures

4 Figure S1. Relationship between $\mathrm{HgT}$ concentrations in filtered and unfiltered samples using different type 5 of membranes, i.e., (A, black dot) untreated Sartobran $(0.2 \mu \mathrm{m}$, cellulose acetate), (A, open dot) acid washed 6 Acropak $(0.2 \mu \mathrm{m}$, polyethersulfone) or (B) acid washed Nuclepore membranes $(0.45 \mu \mathrm{m}$, polycarbonate) 7 often used in the GeOTRACES protocols (Hammerschmidt, C., C. Lamborg, G. Gill and R. Mason. 2010. The 8 GEOTRACES Hg Cookbook Last revised: 6/2/2010).
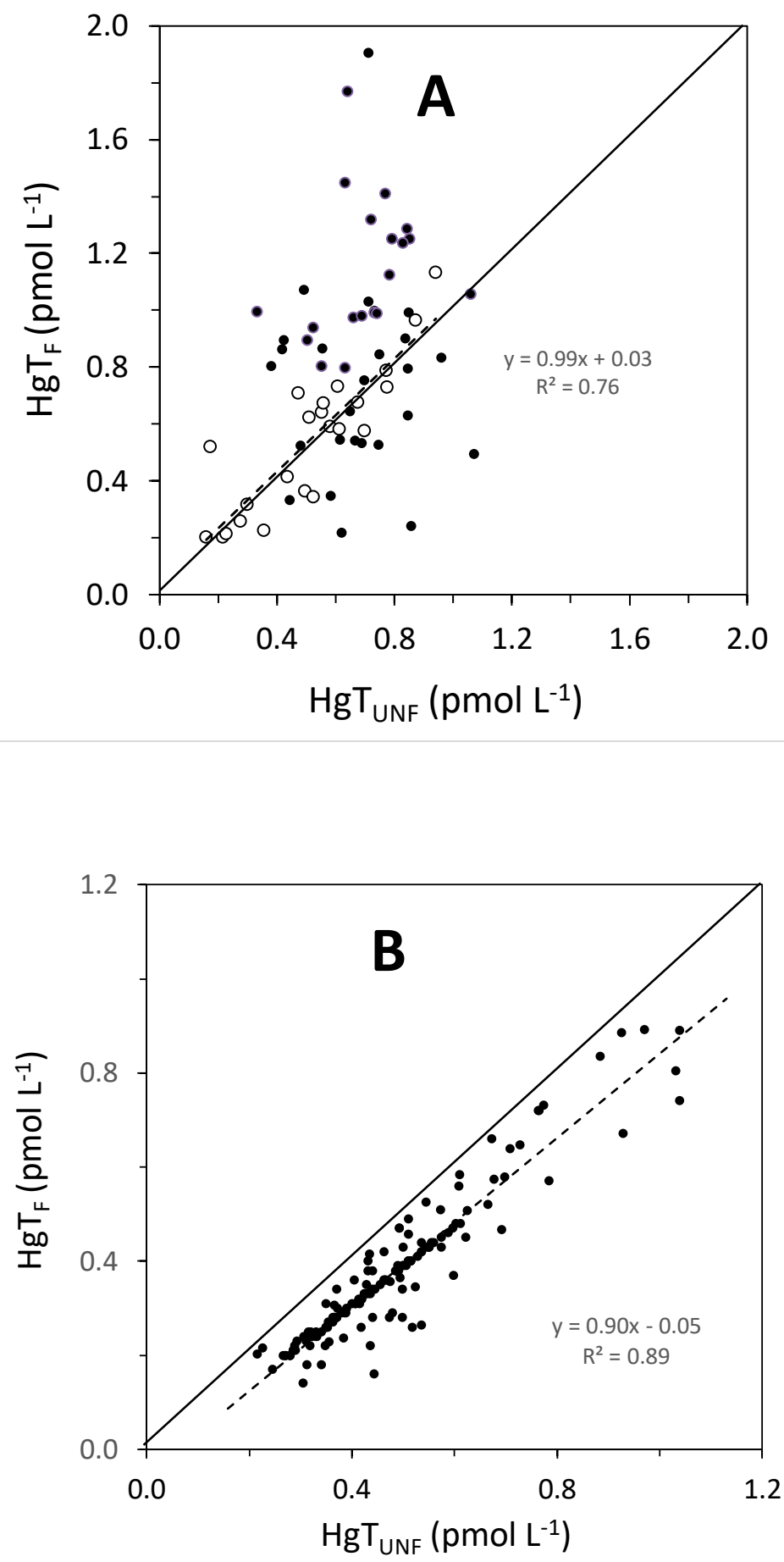
14 Figure S2. (a) Potential temperature $\left(\theta\right.$, in ${ }^{\circ} \mathrm{C}$ ), (b) salinity, (c) oxygen (in $\left.\mu \mathrm{mol} \mathrm{kg}^{-1}\right)$, and (d) silicic acid 15 (in $\mu \mathrm{mol} \mathrm{kg}{ }^{-1}$ ) along 2014 GEOVIDE cruise (GEOTRACES-GA01 section, inset in subplot (a)), from 16 Portugal (right) to Canada (left). The dashed horizontal black line in subplot (d) represents the isopycnal $\sigma 1=32.15 \mathrm{~kg} \mathrm{~m}^{-3}$ (where $\sigma 1$ is potential density referenced to $1000 \mathrm{dbar}$ ), which marks the limit between the upper and lower limbs of the Atlantic Meridional Overturning Circulation (AMOC) at the GEOTRACES-GA01 section (Zunino et al., this issue).
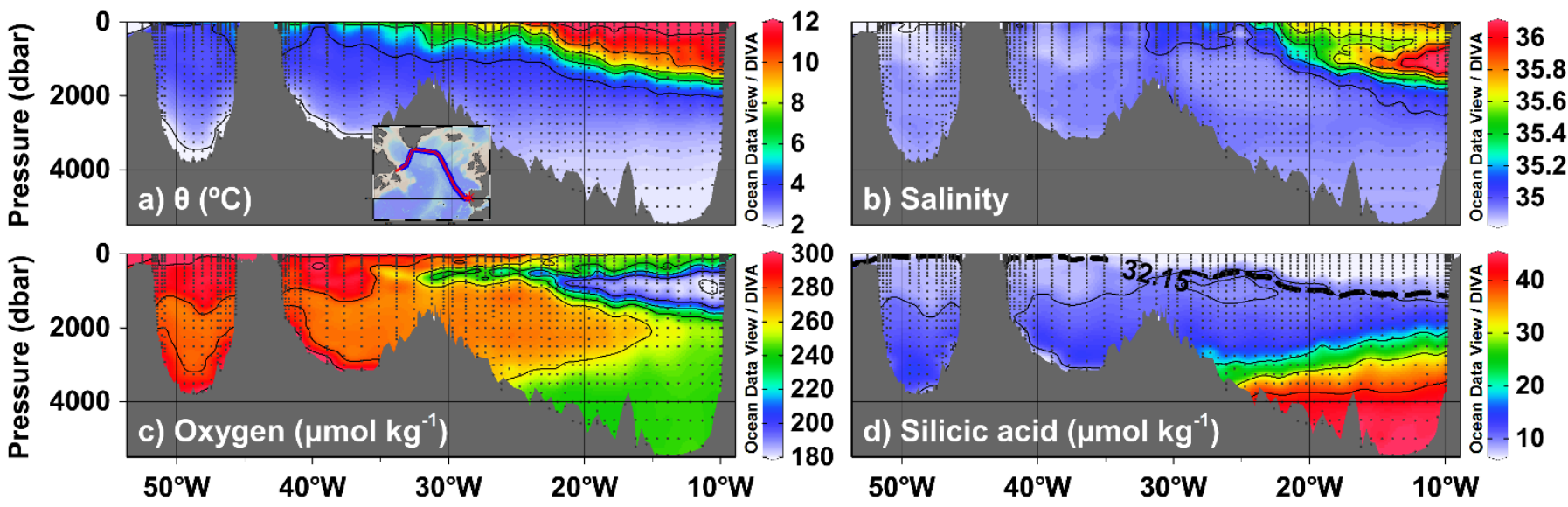

Figure S3. Frequency distribution of $\mathrm{HgT}_{\mathrm{UNF}}$ concentrations measured in the 535 samples collected along the GEOTRACES-GA01 transect.

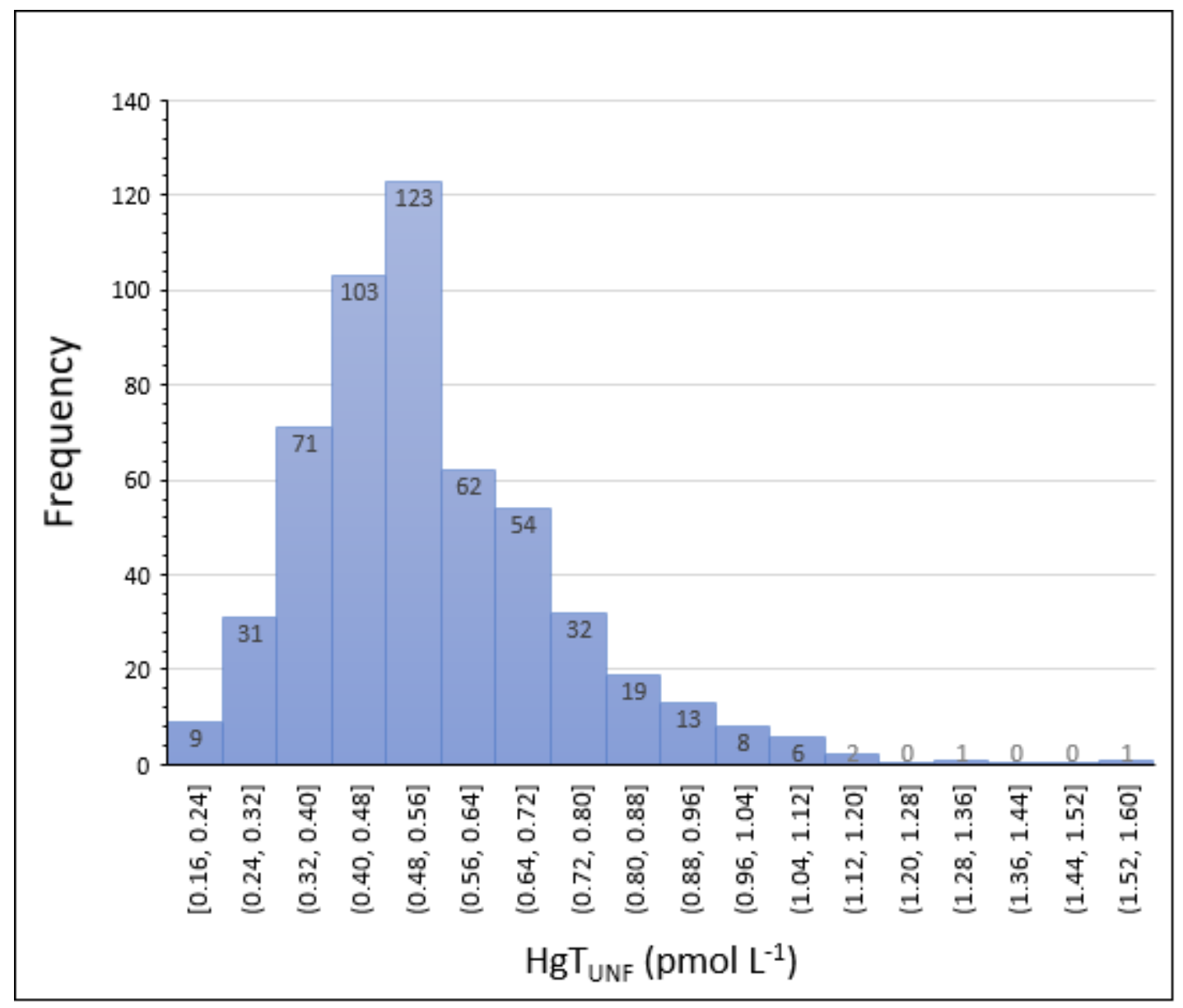


Figure S4. Distribution of $\mathrm{HgT}_{\mathrm{UNF}}$ along the GEOTRACES-GA01 transect modelled by mixing of SWTs.

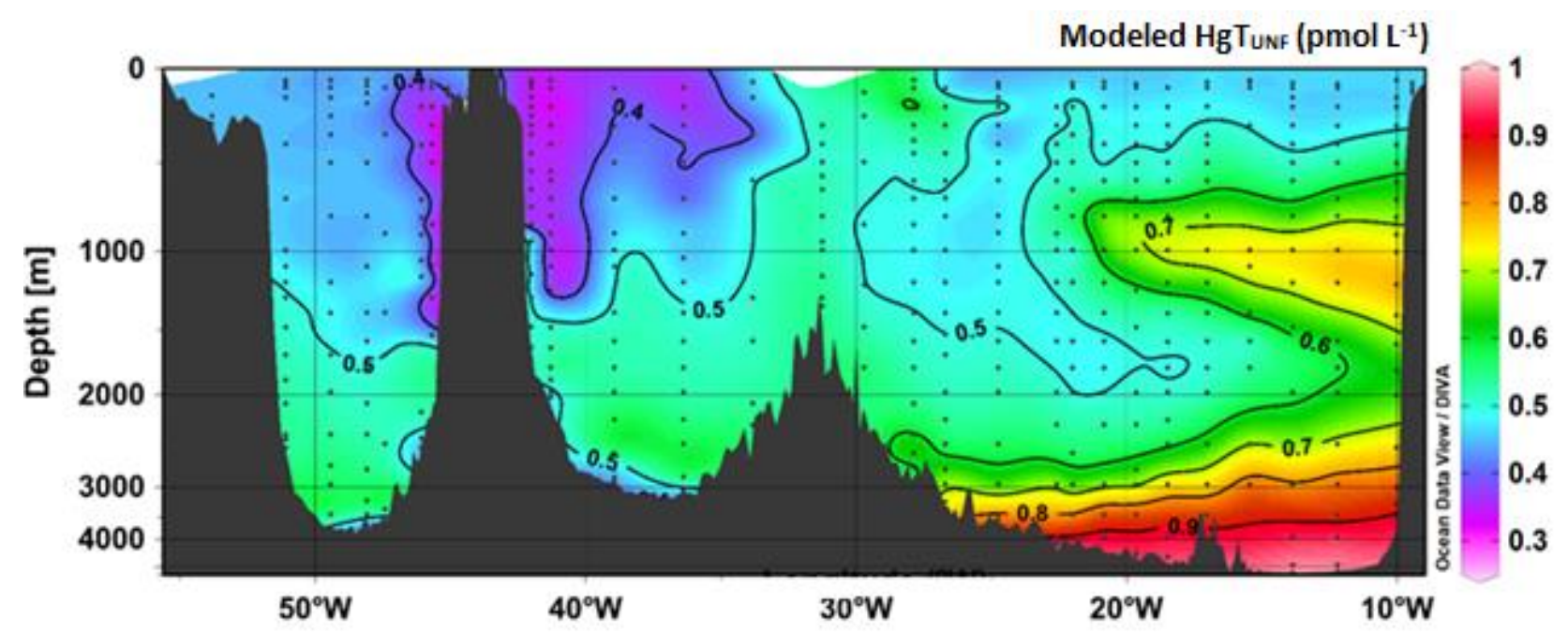

\section{Supplementary tables}

Table S1. Station coordinates along the GEOVIDE cruise (GEOTRACES-GA01 transect). IAP: Iberian Abyssal Plain; ENAB: Eastern North Atlantic basin. Latitude and longitude in decimal degrees.

\begin{tabular}{cccc}
\hline Station & Latitude $\left({ }^{\circ} \mathrm{N}\right)$ & Longitude $\left({ }^{\circ} \mathrm{W}\right)$ & Basin \\
\hline 1 & 40.333 & 10.036 & IAP \\
2 & 40.333 & 09.459 & IAP \\
11 & 40.333 & 12.219 & IAP \\
13 & 41.383 & 13.888 & IAP \\
15 & 42.581 & 15.461 & IAP \\
& & & ENAB \\
17 & 43.780 & 17.031 & ENAB \\
19 & 45.051 & 18.505 & ENAB \\
21 & 46.544 & 19.672 & ENAB \\
23 & 48.039 & 20.848 & ENAB \\
25 & 49.529 & 22.017 & ENAB \\
26 & 50.278 & 22.605 & ENAB \\
29 & 53.020 & 24.752 & ENAB \\
32 & 55.506 & 26.710 & \\
34 & 57.004 & & Iceland Basin \\
36 & 58.207 & 27.879 & Iceland Basin \\
38 & 58.843 & 29.725 & Iceland Basin \\
40 & 59.102 & 31.266 & Iceland Basin \\
42 & 59.363 & 33.828 & Irminger Sea \\
44 & 59.623 & 36.396 & Irminger Sea \\
49 & 59.773 & 38.954 & Irminger Sea \\
& & 41.297 &
\end{tabular}




\begin{tabular}{cccc}
49 & 59.773 & 41.297 & Irminger Sea \\
53 & 59.896 & 43.008 & Irminger Sea \\
60 & 59.799 & 42.013 & Irminger Sea \\
& & & \\
61 & 59.754 & 45.112 & Labrador Sea \\
63 & 59.434 & 45.684 & Labrador Sea \\
64 & 59.072 & 46.089 & Labrador Sea \\
68 & 56.913 & 47.419 & Labrador Sea \\
69 & 55.842 & 48.093 & Labrador Sea \\
71 & 53.692 & 49.433 & Labrador Sea \\
77 & 52.989 & 51.095 & Labrador Sea \\
78 & 51.989 & 53.817 & Labrador Sea \\
\hline
\end{tabular}

34

35 Modeled HgTunf $\left(p m o l ~ L^{-1}\right)$ 
Table S2. Hydrological characteristics (Sal: salinity, Tp: potential temperature, AOU: Apparent Oxygen Utilization, $\sigma_{\theta}$ : potential density), total $\mathrm{Hg}(\mathrm{HgT})$ and anthropogenic $\mathrm{Hg}\left(\mathrm{Hg}_{\text {anth }}\right)$ for the core of Labrador Sea Water (LSW) in the various basin of the North Atlantic Ocean. The core of LSW is defined as water masses with $\sigma_{\theta}$ between 27.74- and 27.82. $\mathrm{Hg}_{\text {Anth }}$ is calculated as the difference between $\mathrm{HgT}$ and

"Remineralized Hg". Due to the slope line $\mathrm{Hg} / \mathrm{P}(1.02 \pm 0.03 \mu$ mole of $\mathrm{Hg}$ per mole of P, according to Ref. 1), the "Remineralized Hg" ( $\mathrm{pmol} \mathrm{L}{ }^{-1}$ ) can be calculated with a Redfield ratio of 140 between $P$ ("Remineralized P") and AOU (Ref. 2). IAP: Iberian Abyssal Plain; ENAB: Eastern North Atlantic basin. Ref. 1: Lamborg C.H., Hammerschmidt C.R., Bowman K.L., Swarr G.J., Munson K.M., Ohnemus D.C., Lam P.J., Heimbürger L.-E., Rijkenberg M.J.A., Saito M.A. 2014. A global ocean inventory of anthropogenic mercury based on water column measurements. Nature, 512, 65-68. Ref. 2: Minster, J.-F., and Boulahdid, M.: Redfield ratios along isopycnal surfaces---a complementary study. Deep Sea Res., 34, 1981-2003, 1987.

\begin{tabular}{|c|c|c|c|c|c|c|c|c|}
\hline Station & $\begin{array}{l}\text { Depth } \\
\text { (m) }\end{array}$ & Sal & $\begin{array}{c}\mathrm{T} p \\
\left({ }^{\circ} \mathrm{C}\right)\end{array}$ & $\begin{array}{c}\text { AOU } \\
\left(\mu \mathrm{mol} \mathrm{L}^{-1}\right)\end{array}$ & $\sigma_{\theta}$ & $\begin{array}{c}\mathrm{HgT}_{\mathrm{UNF}} \\
\left(\mathrm{pmol} \mathrm{L}^{-1}\right)\end{array}$ & $\underset{\left(\mathrm{pmol} \mathrm{L}^{-1}\right)}{\mathrm{Hg}_{\text {Anth }}}$ & Basin \\
\hline 15 & 1582 & 35.03 & 4.30 & 58.3 & 27.78 & 0.68 & 0.25 & IAP \\
\hline 15 & 1779 & 35.01 & 3.99 & 60.4 & 27.79 & 0.69 & 0.30 & IAP \\
\hline 19 & 1582 & 34.94 & 3.89 & 51.1 & 27.75 & 0.54 & 0.17 & ENAB \\
\hline 19 & 1779 & 34.93 & 3.59 & 51.6 & 27.77 & 0.55 & 0.17 & ENAB \\
\hline 19 & 1975 & 34.92 & 3.34 & 51.8 & 27.79 & 0.56 & 0.18 & ENAB \\
\hline 19 & 2221 & 34.92 & 3.14 & 53.5 & 27.81 & 0.57 & 0.18 & ENAB \\
\hline 21 & 1482 & 34.99 & 4.34 & 66.5 & 27.75 & 0.53 & 0.13 & ENAB \\
\hline 21 & 1976 & 34.92 & 3.44 & 66.7 & 27.78 & 0.59 & 0.21 & ENAB \\
\hline 21 & 2269 & 34.92 & 3.16 & 69.6 & 27.81 & 0.56 & 0.17 & ENAB \\
\hline 23 & 1385 & 35.01 & 4.50 & 67.3 & 27.74 & 0.67 & 0.27 & ENAB \\
\hline 23 & 1582 & 34.94 & 3.96 & 63.9 & 27.75 & 0.73 & 0.25 & ENAB \\
\hline 23 & 1778 & 34.92 & 3.67 & 64.2 & 27.76 & 0.58 & 0.21 & ENAB \\
\hline 23 & 1974 & 34.92 & 3.44 & 66.2 & 27.78 & 0.62 & 0.24 & ENAB \\
\hline 23 & 2269 & 34.91 & 3.15 & 68.9 & 27.81 & 0.70 & 0.26 & ENAB \\
\hline 25 & 1580 & 34.92 & 3.82 & 60.0 & 27.74 & 0.60 & 0.25 & ENAB \\
\hline 25 & 1778 & 34.92 & 3.67 & 62.6 & 27.76 & 0.75 & 0.22 & ENAB \\
\hline 25 & 1974 & 34.92 & 3.51 & 65.6 & 27.77 & 0.56 & 0.18 & ENAB \\
\hline 26 & 1384 & 34.92 & 3.79 & 58.6 & 27.74 & 0.69 & 0.34 & ENAB \\
\hline 26 & 1580 & 34.92 & 3.63 & 61.5 & 27.76 & 0.68 & 0.31 & ENAB \\
\hline 26 & 1974 & 34.92 & 3.31 & 65.6 & 27.79 & 0.76 & 0.32 & ENAB \\
\hline 26 & 2268 & 34.92 & 3.08 & 68.3 & 27.82 & 0.73 & 0.33 & ENAB \\
\hline 29 & 1185 & 34.91 & 3.83 & 53.0 & 27.74 & 0.47 & 0.16 & ENAB \\
\hline 29 & 1382 & 34.91 & 3.72 & 57.1 & 27.75 & 0.56 & 0.22 & ENAB \\
\hline 29 & 1581 & 34.92 & 3.57 & 60.9 & 27.77 & 0.49 & 0.22 & ENAB \\
\hline 29 & 1776 & 34.92 & 3.42 & 62.9 & 27.78 & 0.55 & 0.19 & ENAB \\
\hline 29 & 1973 & 34.92 & 3.26 & 64.3 & 27.80 & 0.59 & 0.26 & ENAB \\
\hline 32 & 1383 & 34.91 & 3.73 & 56.4 & 27.75 & 0.55 & 0.22 & Iceland basin \\
\hline 32 & 1532 & 34.92 & 3.64 & 59.2 & 27.76 & 0.49 & 0.29 & Iceland basin \\
\hline 32 & 1680 & 34.92 & 3.55 & 61.1 & 27.77 & 0.69 & 0.30 & Iceland basin \\
\hline 32 & 1974 & 34.92 & 3.29 & 63.4 & 27.80 & 0.72 & 0.31 & Iceland basin \\
\hline 32 & 2218 & 34.93 & 3.10 & 65.9 & 27.82 & 0.63 & 0.29 & Iceland basin \\
\hline 40 & 1282 & 34.94 & 3.70 & 56.8 & 27.77 & 0.63 & 0.30 & Iceland basin \\
\hline 40 & 1578 & 34.93 & 3.42 & 59.0 & 27.79 & 0.58 & 0.24 & Iceland basin \\
\hline 42 & 1185 & 34.90 & 3.72 & 50.2 & 27.74 & 0.42 & 0.22 & Irminger Sea \\
\hline 42 & 1383 & 34.93 & 3.70 & 57.8 & 27.77 & 0.59 & 0.19 & Irminger Sea \\
\hline
\end{tabular}




\begin{tabular}{lllllllll}
42 & 1580 & 34.94 & 3.53 & 59.9 & 27.79 & 0.65 & 0.23 & Irminger Sea \\
42 & 1777 & 34.93 & 3.34 & 61.4 & 27.80 & 0.51 & 0.19 & Irminger Sea \\
42 & 1972 & 34.94 & 3.19 & 62.9 & 27.82 & 0.53 & 0.19 & Irminger Sea \\
44 & 1087 & 34.91 & 3.70 & 50.6 & 27.74 & 0.44 & 0.13 & Irminger Sea \\
44 & 1236 & 34.91 & 3.63 & 55.0 & 27.76 & 0.49 & 0.16 & Irminger Sea \\
44 & 1382 & 34.93 & 3.56 & 58.3 & 27.78 & 0.50 & 0.15 & Irminger Sea \\
44 & 1581 & 34.93 & 3.40 & 60.1 & 27.79 & 0.50 & 0.15 & Irminger Sea \\
44 & 1776 & 34.93 & 3.20 & 61.2 & 27.81 & 0.52 & 0.16 & Irminger Sea \\
49 & 989 & 34.92 & 3.79 & 48.5 & 27.75 & 0.37 & 0.08 & Irminger Sea \\
49 & 1088 & 34.93 & 3.74 & 50.8 & 27.76 & 0.39 & 0.09 & Irminger Sea \\
49 & 1235 & 34.93 & 3.56 & 52.2 & 27.78 & 0.39 & 0.08 & Irminger Sea \\
49 & 1482 & 34.91 & 3.17 & 53.4 & 27.80 & 0.43 & 0.12 & Irminger Sea \\
49 & 1629 & 34.91 & 2.90 & 53.5 & 27.82 & 0.42 & 0.12 & Irminger Sea \\
& & & & & & & & \\
68 & 1382 & 34.86 & 3.42 & 41.5 & 27.74 & 0.37 & 0.15 & Labrador Sea \\
68 & 1677 & 34.92 & 3.57 & 59.2 & 27.77 & 0.46 & 0.13 & Labrador Sea \\
68 & 2463 & 34.92 & 2.89 & 66.4 & 27.82 & 0.50 & 0.14 & Labrador Sea \\
69 & 1381 & 34.86 & 3.40 & 41.3 & 27.74 & 0.36 & 0.15 & Labrador Sea \\
69 & 1580 & 34.89 & 3.53 & 51.9 & 27.75 & 0.44 & 0.15 & Labrador Sea \\
69 & 1776 & 34.92 & 3.54 & 60.3 & 27.77 & 0.49 & 0.15 & Labrador Sea \\
69 & 2071 & 34.92 & 3.26 & 62.8 & 27.80 & 0.52 & 0.15 & Labrador Sea \\
69 & 2365 & 34.92 & 2.99 & 65.0 & 27.82 & 0.51 & 0.16 & Labrador Sea \\
71 & 1383 & 34.91 & 3.64 & 43.9 & 27.75 & 0.46 & 0.14 & Labrador Sea \\
71 & 1679 & 34.92 & 3.49 & 48.1 & 27.78 & 0.49 & 0.14 & Labrador Sea \\
71 & 1974 & 34.92 & 3.21 & 48.6 & 27.80 & 0.51 & 0.16 & Labrador Sea \\
71 & 2366 & 34.92 & 2.87 & 49.1 & 27.82 & 0.54 & 0.18 & Labrador Sea \\
& & & & & & & & \\
\hline
\end{tabular}

